\title{
Field of View and Skill Training of Table Tennis Teaching Based on Virtual Environment Technology
}

\author{
https://doi.org/10.3991/ijet.v11i11.6240
}

$\mathrm{Li} \mathrm{Li}$

Jilin Engineering Normal University, Jilin, China

\begin{abstract}
Focusing on the topic of improving the table tennis teaching quality with the visual field training, the test is to grab images repeatedly by using a camera in a speed of $119 \mathrm{~s}$, and the final result shows that it gets the correct hitting points on racket coordinating with correct racket angles, which verifies the effectiveness of the visual field training. Finally, it's concluded that the visual field training can improve the athletes' abilities of focus and judgment, and enhance their exercise stability.
\end{abstract}

Index Terms - field of view, skill training, table tennis teaching, virtual environment fitting technology

\section{INTRODUCTION}

Modern information technology is integrating with the whole process of education teaching, and is changing the process and pattern of original education teaching gradually[1]. Virtual reality is a kind of new computing environment, of which one of the critical characteristics is interactivity. Intelligent Virtual Environment is an outcome of Artificial Intelligence and Virtual Environment. Due to its features: scalable, autonomous, proactive, reconfigurable, agile, the Virtual Environment Systems (VES) has been the most interesting AI technique[2-3].

Virtual Reality is a new research paradigm in psychology research area; it has some characteristics such as multi-perception, immersive and interaction. Virtual reality solves the problem that perception and action separating, leads the perception and action study more natural, improves the study ecological validity, and holds great promise for the study of perception- action. In decades, researches about the relationship between perception and action in baseball, handball and tennis found that, athletes can make exact interceptive actions in virtual environment. In future, more studies should be focused on not the relationship between perception and action, but its mechanisms by using ERP / f MRI technical, aiming to provide theory and practice guidance to athletes' training and competing [4-5].

Table tennis is regarded as "the National Game" in our country, it is just like a social symbol and it plays an important role in our people's opinion. In our country, table tennis has gained great achievement and occupied an important position cannot be changed by other countries. As the most important country in the field of table tennis, we have the responsibility and obligation to undertake the development task of table tennis [6]. In the process of the development of table tennis, we have to clearly recognize that the occupation and monopolization of a sports item is not good to the development of this item. To an item of sport itself, there is no boundary, it should be universal and service the people all over the world. To realize the sustained and stable development of table tennis around the world, we have to pay attention to improve the awareness of its consciousness of culture.

Virtual Environment (VE), vivid and realistic environment in computers, usually refers to the multiple dimensions information space, in which computers are used as the core equipment and modern high-tech techniques are applied so that many senses such as vision, hearing and touching are integrated together and characteristics of Time and Space are possessed and Virtual Reality technology is embodied concretely. Virtual environment, which can be the nonexistent environment at present, can preview the future works to the people in advance; which can also be the ultra-limit environment that the people cannot arrive because of limit of time, space and physical strength, ability, can control and display those objective things vividly, directly, figuratively and racially; which can take place of the expensive realistic environment, economize the time and space and decline the cost. In brief, in virtual environment, the people can interact mutually with an object in virtual environment naturally and affect each other conveniently, thus the feeling and the experience of real world can be obtained through virtual environment [7-9]. Virtual environment base on the real world, surmount it, and then intelligent it and predict the future scientifically.

This paper mainly studies the application of the virtual environment fitting technology in skill training of table tennis teaching from five parts. The first section is the preface which introduces the study purpose, background, content and methods of this paper. The second section is the overview part. It summarizes the related researches of the virtual environment fitting technology in skill training of table tennis teaching, both at home and abroad. The third section is an important part of this article which dissects the necessity and viability to establish a virtual environment fitting model in the table tennis teaching. It reveals the interdependence and promoting relationships between the virtual environment fitting technologies in the table tennis teaching. It introduces the basic algorithm in the table tennis teaching. The fourth section makes a detailed mode of the virtual environment fitting technology in the table tennis teaching. The experiment is done in this part. The fifth section discusses the comparison result and data obtained in the fourth part. 


\section{METHOD AND ALGORITHM}

Some teachers are traditional and teach according to the original PE principle without establishing the thought of lifelong education. So many students consider that only the weak students need physical exercise and training, causing the inactivity of many students in PE lessons and little interest in table tennis. In addition, the students cannot have a thorough understanding of the knowledge and learn enough skills with few table tennis lessons. Some teachers also cannot teach the comprehensive knowledge with the limit of class our, causing the decrease of teaching quality. In the teaching process of table tennis, because the learning behavior and process cannot be controlled well, the learning results are different; many students cannot master some motion or skills of kind of ball. The training time and amount of exercise are limited by the large amount of students. So the students are not familiar with some detail, causing not enough interest of table tennis. Consequently, in the teaching process, the students should be taught in accordance with their aptitude and knowledge of various fields should be taught and connected separately. Then the students can understand the principle of table tennis, master real skills and get more confidence and happiness in sports.

With a small volume, table tennis requires athletes with attention, stability and judgment. Firstly, in nervous competition process, the athletes should control psychology themselves well and deal with the pressure of the opponents and outside disturbance, making attention training necessary. The visual field training can make the athletes effectively focus and easily devote themselves to the competition in competitions and other situations. Meanwhile, the visual field training can effectively exercise stability and judgment. It can make athletes judge the position and direction of the ball more accurately and strike back better. Also it can improve the stability of the athletes and make them calmly analyze the situation and strike back better in an unexpected situation. So the visual field training has a significant meaning in table tennis teaching. And the training and learning of it can bring much fun in sports of table tennis.

The basic algorithm is shown in the following equations:

$$
\varphi_{j i}\left(\mu_{j}\right)=\exp \left(\frac{-\left(\mu_{j}-C_{j i}\right)^{2}}{b_{j i}{ }^{2}}\right) \text {, for } i=1,2, \mathrm{~K}, H
$$

In this space, the mth multidimensional receptive-field function is defined as

$$
\Phi_{m}(\mu)=\prod_{j=1}^{L} \varphi_{j i}\left(\mu_{j}\right) \text {, for } m=1,2, \mathrm{~K}, N
$$

The function can be written in a vector notation as

$$
\Phi(\mu, C, b)=\left[\Phi_{1}, \Phi_{m} \ldots, \Phi_{N}\right]^{T}
$$

where $C=\left[C_{11}, \ldots, C_{L 1}, C_{12}, \ldots, C_{L 2}, \ldots, C_{1 H}, \ldots C_{L H}\right]^{T}$, and $b=\left[b_{11}, \ldots, b_{L 1}, b_{12}, \ldots, b_{L 2}, \ldots, b_{1 H}, \ldots b_{L H}\right]^{T}$.

The weight memory space with $\mathrm{N}$ components can be expressed in a vector as

$$
W=\left[W_{1}, W_{m}, \ldots, W_{N}\right]^{T}
$$

The activated weights in weight memory space, which can be written in a vector form as

$$
y=W^{T} \Phi(\mu)
$$

The state variables and the desired values can be defined as follows:

$$
z_{1}=x_{1}-y_{d}
$$

and

$$
z_{2}=x_{2}-\alpha_{1}
$$

The following tracking error dynamics is shown as:

$$
\dot{z}_{1}=\dot{x}_{1}-\dot{y}_{d}=x_{2}-\dot{y}_{d}=z_{2}+\alpha_{1}-\dot{y}_{d}
$$

The first derivative of the Lyapunov function can be written as

$$
\begin{aligned}
& \dot{V}_{1}=z_{1}^{T} \dot{z}_{1}=z_{1}^{T}\left(\dot{x}_{1}-\dot{y}_{d}\right)=z_{1}^{T}\left(\dot{x}_{1}-\dot{y}_{d}\right)=z_{1}^{T}\left(x_{2}-\dot{y}_{d}\right) \\
& =z_{1}^{T}\left(z_{2}+\alpha_{1}-\dot{y}_{d}\right)=-\lambda_{1} z_{1}^{T} z_{1}+z_{1}^{T} z_{2}
\end{aligned}
$$

From (2) and (6), it can be obtained:

$$
\dot{z}_{2}=\dot{x}_{2}-\dot{\alpha}_{1}=-M^{-1} C x_{2}-M^{-1}\left(G_{g}+d\right)+M^{-1} \tau-\dot{\alpha}_{1}
$$

$\tau$ is selected as

$$
\tau=-\lambda_{2} z_{2}-z_{1}-F
$$

Then we can get:

$$
\begin{aligned}
& V_{2}=V_{1}+\frac{1}{2} z_{2}^{T} M z_{2} \\
& \dot{V}_{2}=\dot{V}_{1}+\frac{1}{2} z_{2}^{T} M \dot{z}_{2}+\frac{1}{2} \dot{z}_{2}^{T} M z_{2}+\frac{1}{2} z_{2}^{T} \dot{M} z_{2} \\
& =-\lambda_{1} z_{1}^{T} z_{1}+z_{1}^{T} z_{2}+z_{2}^{T} M\left(\dot{x}_{2}-\dot{\alpha}_{1}\right)+z_{2}^{T} C z_{2} \\
& =-\lambda_{1} z_{1}^{T} z_{1}+z_{1}^{T} z_{2}+z_{2}^{T}\left(-C x_{2}+C z_{2}+\tau\right. \\
& \left.-M \dot{\alpha}_{1}-\left(G_{g}+d\right)\right) \\
& =-\lambda_{1} z_{1}^{T} z_{1}+z_{1}^{T} z_{2}+z_{2}^{T}(f+\tau)-z_{2}^{T}\left(G_{g}+d\right) \\
& \dot{V}_{2}=-\lambda_{1} z_{1}^{T} z_{1}-\lambda_{2} z_{2}^{T} z_{2}+z_{2}^{T}(f-F)-z_{2}^{T}\left(G_{g}+d\right)
\end{aligned}
$$

The ideal weight $\mathrm{W}$ from (10) and expressed as

$$
F=W^{T} \Phi(\mu)
$$

Define the estimate of the value of (11) as

$$
\hat{F}=\hat{W}^{T} \Phi(\mu)
$$

It's provided by the adaptive weight law. So estimation error of the weight is

$$
\tilde{W}=W-\hat{W}
$$

The positive values Wmax as follows:

$$
\|W\|_{F} \leq W_{\max }
$$

The adaptive weights law is defined as

$$
\dot{\hat{W}}=-k G\left\|z_{2}\right\| \hat{W}-z_{2}^{T} G \Phi(\mu)
$$

By differentiating the yields of $\mathrm{V}$, we obtain 


$$
\begin{aligned}
\dot{V}= & \dot{V}_{2}+\operatorname{tr}\left\{\tilde{W}^{T} G^{-1} \dot{\tilde{W}}\right\} \\
= & -\lambda_{1} z_{1}^{T} z_{1}-\lambda_{2} z_{2}^{T} z_{2}+z_{2}^{T}(f-F) \\
& -z_{2}^{T}\left(G_{g}+d\right)+\operatorname{tr}\left\{\tilde{W}^{T} G^{-1} \dot{\tilde{W}}\right\} \\
= & -\lambda_{1} z_{1}^{T} z_{1}-\lambda_{2} z_{2}^{T} z_{2}+z_{2}^{T}(f-\hat{F}+\hat{F}-F) \\
& -z_{2}^{T}\left(G_{g}+d\right)+\operatorname{tr}\left\{\tilde{W}^{T} G^{-1} \dot{\tilde{W}}\right\}
\end{aligned}
$$

The unknown function $\mathrm{f}$ is such that

$$
\|f-\hat{F}\| \leq \varepsilon_{0}
$$

The formula (20) can be written as

$$
\begin{aligned}
& \dot{V}=-\lambda_{1} z_{1}^{T} z_{1}-\lambda_{2} z_{2}^{T} z_{2}+z_{2}^{T} \\
& \left(f-\hat{F}+\hat{W}^{T} \Phi(\mu)-W^{T} \Phi(\mu)\right) \\
& -z_{2}^{T}\left(G_{g}+d\right)+\operatorname{tr}\left\{\tilde{W}^{T} G^{-1} \dot{\tilde{W}}\right\} \\
& =-\lambda_{1} z_{1}^{T} z_{1}-\lambda_{2} z_{2}^{T} z_{2}+z_{2}^{T}(f-\hat{F}) \\
& -z_{2}^{T} \tilde{W}^{T} \Phi(\mu) \\
& -z_{2}^{T}\left(G_{g}+d\right)+\operatorname{tr}\left\{\tilde{W}^{T} G^{-1} \dot{\tilde{W}}\right\} \\
& =-\lambda_{1} z_{1}^{T} z_{1}-\lambda_{2} z_{2}^{T} z_{2} \\
& +z_{2}^{T}(f-\hat{F})-z_{2}^{T}\left(G_{g}+d\right) \\
& +\operatorname{tr}\left\{\tilde{W}^{T}\left(G^{-1} \dot{\tilde{W}}_{-} z_{2}^{T} \Phi(\mu)\right)\right\}
\end{aligned}
$$

The usual visual field training methods in table tennis teaching, including the focus expansion training method and the focus moving training method, are different in process, but providing help for the table tennis teaching. Firstly, the former is as follows: the eyes are focused in the center of the table tennis, decreasing the time and frequency of blink; the position and movement of the head are kept still and the eyes rotational quickly, and the above exercise is taken by the way of uptown, left-right, firstquarter and last-quarter. Some reversal exercise can be taken with certain proficiency. Each movement should be kept above ten seconds, thus expanding the horizon, focusing, activating brain and improving reaction. The longtime insistence can increase the advantage and achievability, thus making people happy and healthy while having fun. Secondly, the latter is as follows: the sight line should follow the movement of table tennis; in the moving process the eyesight should be focused at a point quickly and duly stay still; the frequency of blink should be decreased. Insisting focusing in the moving process for a long time can make the point and the line clear, improve the stability and make themselves quickly judge and strike back in a short time.

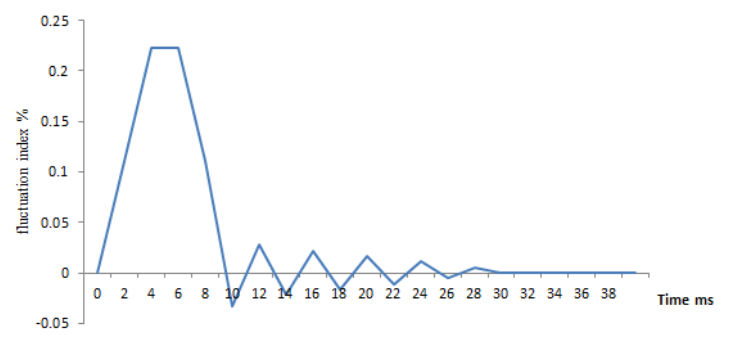

Figure 1. Volume fluctuation of the tennis ball after hitting.
The visual field training is a preparing and assistant training, but occupying a very important position in table tennis and determining the result of the competition to some content. So the visual field training should be concerned to improve the teaching quality and motor skills in process of table tennis teaching.

\section{EXPERIMENT RESULT}

To verify the method of this paper reliable, experiments were well conducted. The monocular vision system was made up of a Lenovo computer and a camera GC660C, which could grab images in a speed of 119 s. First, we captured some images to detect the corners, where the poses of the racket were different. As shown in Fig. 2, some extreme racket poses could be extracted via the method of this paper. From the results, it could be seen that if the thresholds were not computed well using the method in this paper, the method doesn't work well. Secondly, randomly selecting multiple images from a video, we attempted to extract the corners from those images via the two methods. We could see that there were some errors in the two methods, and some errors were relatively large, a special reason causing the result is that some racket poses are so extreme that the results aren't ideal. Other reasons include that via the method we didn't change the thresholds and the centers of the corners sometimes were in the middle of two pixels. Finally, we rotated the racket 1800 about the $\mathrm{W}$-axis, the beginning position was that the racket was parallel to the $\mathrm{Xv}, \mathrm{Yv}$ plane, only the pitch angle changed from 00 to 1800 , The result which showed that we got the corners coordinates successful is shown in the Fig. 3.

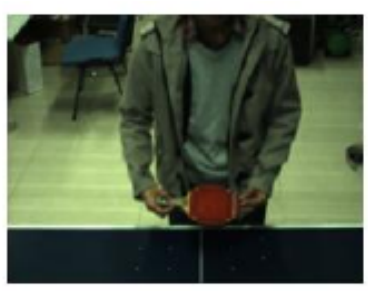

a)

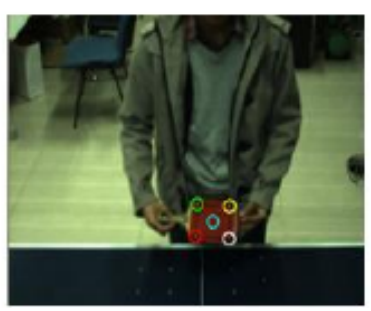

c)

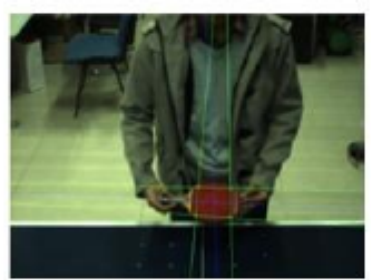

e)

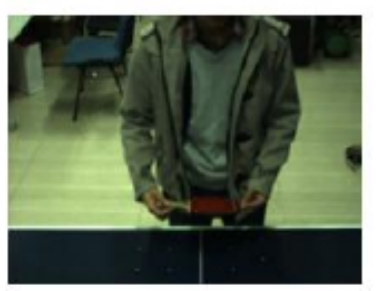

b)

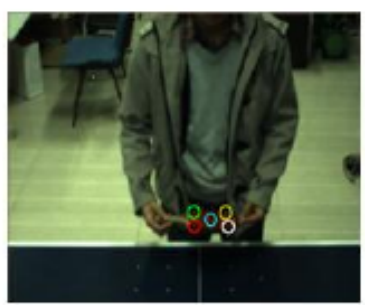

d)

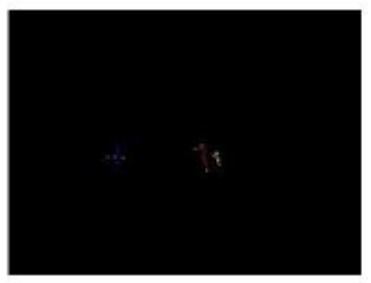

f)
Figure 2. The result 


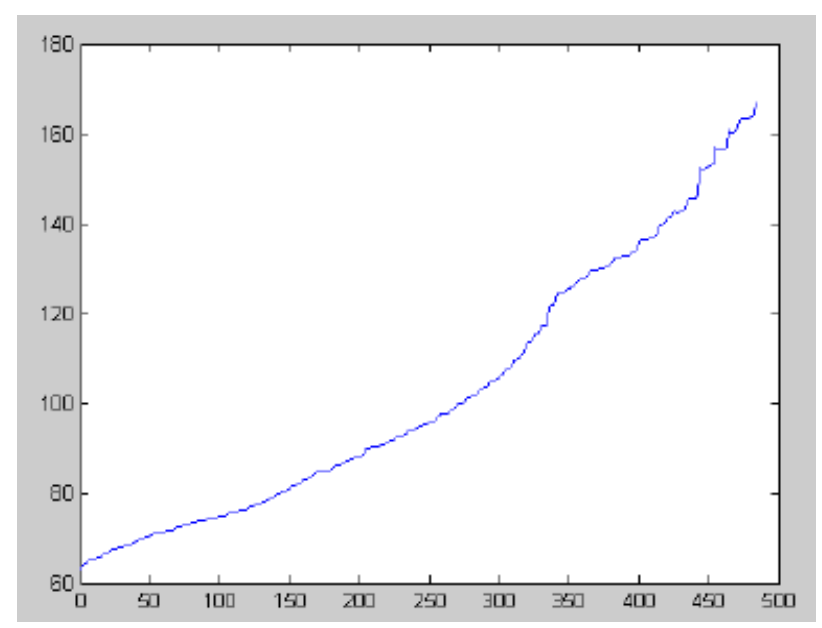

Figure 3. The angle which can detect the corners

Under the visual environment, the point training can be realized. The point training can be divided into straight line to point training method and diagonal line to point training method, and they can transform and complement each other. When one hits by straight line, the other will hit back by straight line or diagonal line, or transform the way deliberately and randomly hit back without a fixed impact point. The students should have the acquired reflex trained constantly by combining straight line to point training method with diagonal line to point training method. Then they can deal with the situation that the impact points are different. Although the training method takes time, it can take effect by combining with other skills and cooperating with partners, and the advantage of point training method can be found.

\section{CONCLUSION}

The table tennis lessons are set for the purpose of strengthening the body of students, motivating the interest to sports, increasing activity and improving health. Table tennis is more and more popular, although there are some problems in the teaching process. In the work, the problems are analyzed and the methods of visual field and skill training are proposed. E.g. the visual field training metical contains the focus expansion training metical and moving training method and the skill training method contains the point training method, three deep and two drop training method, single or dead line training method, a combination method of multiple skills and mental quality training method.

The visual field training can make the athletes effectively focus and easily devote themselves to the competition in competitions and other situations. Meanwhile, the visual field training can effectively exercise stability and judgment. It can make athletes judge the position and direction of the ball more accurately and strike back better. Also it can improve the stability of the athletes and make them calmly analyze the situation and strike back better in an unexpected situation. So the visual field training has a significant meaning in table tennis teaching. And the training and learning of it can bring much fun in sports of table tennis.

With the effort of the education department, the teachers and the students, the teaching process of table tennis will be more and more abundant and more and more students will be interested in table tennis and change the attitude to sports and table tennis. Meanwhile, the teachers will become more and more and the table tennis teaching quality will be increased, making contributions to the improvement of the national constitution and the development of the comprehensive talents.

\section{REFERENCES}

[1] H. Jing, "The Study on the Impact of Data Storage from Accounting Information Processing Procedure," International Journal of Database Theory and Application, vol. 8, no.3, pp. 323-332, June 2015. https://doi.org/10.14257/ijdta.2015.8.3.28

[2] D. Jiang, Z. Xu, and Z. lv, "A multicast delivery approach with minimum energy consumption for wireless multi-hop networks," Telecommunication Systems, vol. 62, no.4, pp. 1-12, August 2015.

[3] Y. Zhou, "Application of Automatic Choreography Software Based on Virtual Technology in the Gymnastics Teaching," International Journal of Emerging Technologies in Learning, vol. 11, no.5, pp. 39-44, May 2016. https://doi.org/10.3991/ijet.v11 i05.5692

[4] J. Shen, X. Guan, and Z. Liao, "Three-dimensional numerical simulation of racket-holding hand of tennis players", Ching $J$ Sports Med, Vol 33, No 5, May. 2014.

[5] M. Viceconti, C. Zannoni, D. Testi, et al., "A new method for the automatic mesh generation of bone segments from CT data," $J$ Med Eng Technol, vol. 23, no.2, pp. 77-81, 1999. https://doi.org/10.1080/030919099294339

[6] D. Ulrich, B. V. Rietbergen, A. Laib, et al., "Load transfer analysis of the distal radius from in-vivo high-resolution CTimaging," J Biomech, vol. 32, no.8, pp. 821-828, 2009. https://doi.org/10.1016/S0021-9290(99)00062-7

[7] W. Pistoia, B.V. Rietbergen, E. M. Lochmüller, et al., "Estimation of distal radius failure load with micro-finite element analysis models based on three-dimensional peripheral quantitative computed tomography images," Bone, vol. 30, no.6, pp. 842-848, 2002. https://doi.org/10.1016/S8756-3282(02)00736-6

[8] E. J. Lee, et al., "Recognizing pedestrian's unsafe behaviors in farinfrared imagery at night," Infrared Physics \& Technology, 76, pp. 261-270, 2016. https://doi.org/10.1016/j.infrared.2016.03.006

[9] Y. Liu, J. Shao, and Y. Yue, "The Application of Improved GG Clustering Algorithm in View-irrelevant Behavior Recognition," Atlantis Press, 2015. https://doi.org/10.2991/ameii-15.2015.4

\section{AUTHOR}

Li Li is with Jilin Engineering Normal University, Jilin, China (363219209@qq.com).

Submitted 09 September 2016.Published as resubmitted by the author 13 October 2016. 